\title{
Study of feasibility of portal dosimetry in comparison with ImatriXX 2-D array system for IMRT and Rapid arc patient specific QA.
}

\author{
Sajeev Surendran ${ }^{1,2}$, Durga Prasada Rao ${ }^{2}$, Swapna Lilly Cyriac ${ }^{1}$, \\ Jayesh kunnanchath ${ }^{3}$, Saroj Kumar das majumdar ${ }^{3}$ \\ ${ }^{1}$ Kims Pinnacle cancer care and research center, Trivandrum, Kerala, India. \\ ${ }^{2}$ Department of Nuclear Physics, Andhra University, vizag, Andhrapradesh, India. \\ ${ }^{3}$ Department of Oncology, Salmaniya Medical Complex, Bahrain.
}

\begin{abstract}
This study aimed to compare the Varian portal dosimetry system and the ImatriXX 2-D array system of IBA dosimetry for IMRT and Rapid arc patient specific quality assurance (QA). Fifteen cases of IMRT and fifteen cases of Rapid arc plans were selected for the patient specific QA study using portal dosimetry and ImatriXX 2-D array system. Planar dose comparison was carried out with gamma criteria of $3 \%-3 \mathrm{~mm}$ \{dose difference and distance-to-agreement (DTA)\}. For portal dosimetry system area gamma, average gamma and maximum gamma were analyzed and for the ImatriXX 2-D array system, the $\%$ of pixels passing the $3 \%-3 \mathrm{~mm}$ gamma was chosen.

In case of IMRT, the portal dosimetry mean values of area gamma, average gamma and maximum gamma were $1.01,0.48$ and 1.72 with standard deviation values of $0.38,0.05$ and 0.20 and for Rapid arc the corresponding values were 2.01, 0.48 and 1.72 with standard deviation of $0.54,0.07$ and 0.29 . With the ImatriXX 2-D array system, on an average $99.22 \%$ of the pixels passed the criteria of $3 \%-3 \mathrm{~mm}$ with standard deviation of 0.58 for IMRT. For rapid arc, the average value was $97.14 \%$ with a standard deviation of 1.26 . The results showed that both the systems can be use for patient specific QA measurements for IMRT and rapid arc. Portal dosimetry as well as ImatriXX measurements showed significant difference in gamma results of IMRT vs. rapid arc with pvalue less than 0.05 . Compared to ImatriXX 2-D array system, the portal dosimetry values were more consistent. The portal dosimetry QA is less time consuming and in case of IMRT individual field verification is easier compared to ImatriXX 2-D array system.
\end{abstract}

Keywords: - Intensity modulated radiotherapy, Rapid arc, ImatriXX, Portal dosimetry, $Q A$

\section{INTRODUCTION}

Intensity modulated radiotherapy (IMRT) and rotational rapid arc therapy (RA) are the most common radiotherapy techniques used to treat the cancer. Compared to the olden techniques like conventional or 3-D conformal radiotherapy, the IMRT and rapid arc are giving uniform desired dose distribution to the target volume with adequate sparing of the near-by critical structures. The portal dosimetry system and 2-D array system are widely using as relative dosimetric detectors for the planar dose comparison of treatment planning system (TPS) vs. measured dose, because of their consistency in results, less time consuming, and easy to use[1,2]. There are many studies regarding the characteristics of these detectors, uses of these detectors for patient specific quality assurance (QA) and machine QA (3-9). Because of the modifications in the design of detectors, their characteristics as well as the introduction of new techniques in radiotherapy, feasibility of these detectors to use as an ideal dosimeter for patient specific QA of IMRT and rapid arc is still a subject of controversy[10, 11]. This study aimed to compare the Varian portal dosimetry system and the ImatriXX 2-D array system of IBA dosimetry for IMRT and rapid arc patient specific QA

\section{MATERIALS AND METHODS}

The aSi1000 electronic portal imaging device (EPID) is a flat panel X-ray imager with large area active matrix readout structure, made up of phosphor or photo conductor. Here each pixel in the matrix consists of aSi photo cathode and a thin film transistor (TFT). The associated electronics with the TFT switches enables the charge capture readout. The image acquisition system with fast readout electronics enable up to 30 frames per second in aSi 1000 EPID. The resolution of aSi 1000 EPID is upto $0.39 \mathrm{~mm}$. The ImatriXX 2-D array system consists of 1020 parallel plate ion chamber arranged in a 32x32 grid, with an inter detector spacing of 7.619 $\mathrm{mm}$. Each detector is having a diameter of $4.5 \mathrm{~mm}$, height $5 \mathrm{~mm}$ and chamber volume $0.02 \mathrm{cc}$. The measurements were performed in the Varian Clinac iX linear accelerator. It is having a capability to deliver IMRT as well as rapid arc plans. 
In this work fifteen cases of dynamic IMRT and fifteen cases of double arc Rapid arc plans were selected for the patient specific QA study using portal dosimetry and ImatriXX 2-D array system. Planar dose comparison was carried out with gamma criteria of 3\% $-3 \mathrm{~mm}$ \{dose difference and distance-to-agreement (DTA) . The portal dosimetry work flow and ImatriXX 2-D array work flow is as shown in figure1 and figure 2. For portal dosimetry system area gamma, average gamma and maximum gamma were analyzed and for the ImatriXX 2-D array system, the \% of pixels passing the 3\%-3mm gamma were chosen.
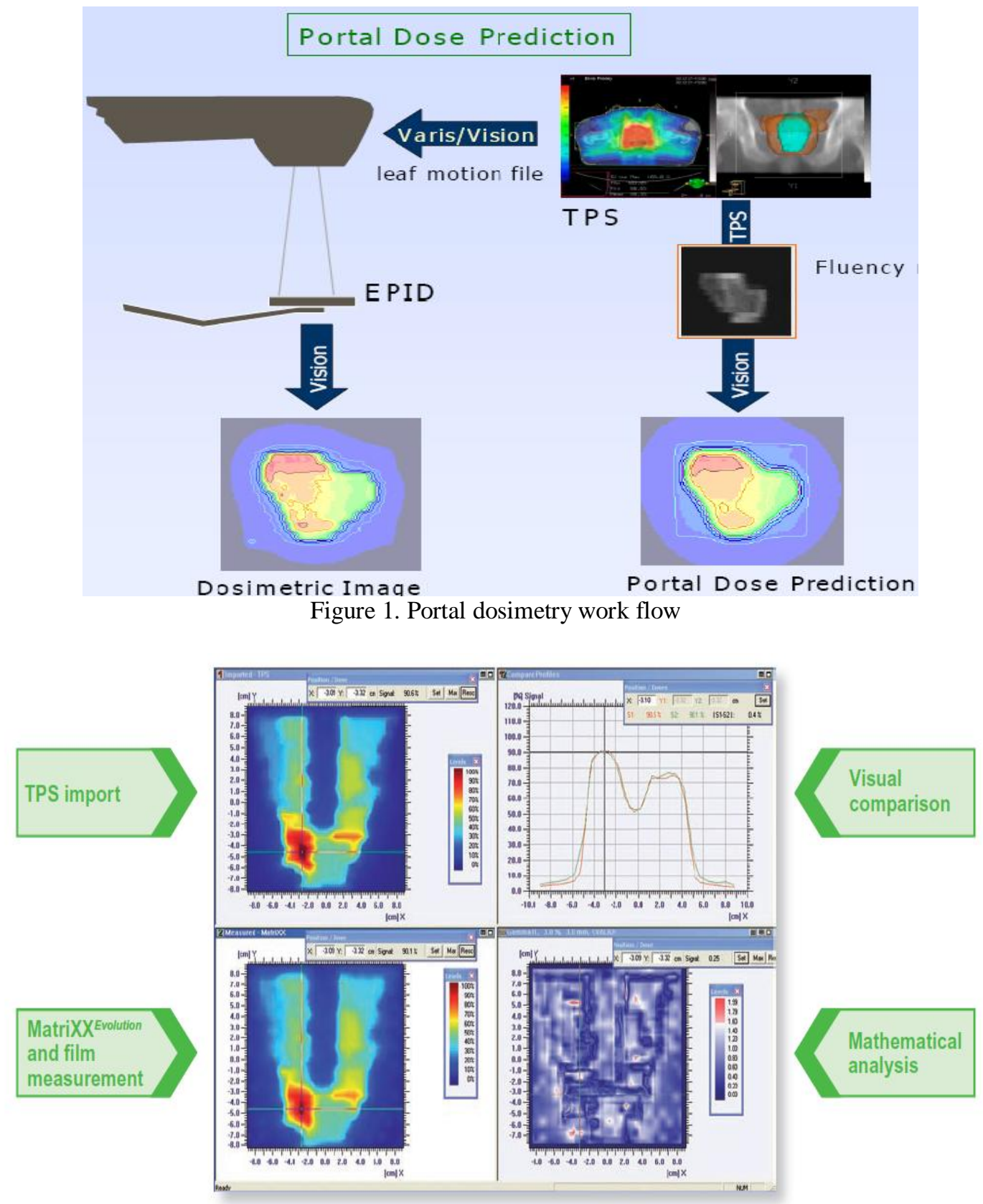

Figure2. ImatriXX 2-D array system - work flow

\section{RESULTS AND DISCUSSION}

The gamma results (\% of dose difference and distance to agreement) for fifteen IMRT and fifteen rapid arc cases were measured. Portal dosimetry results such as area gamma, average gamma and maximum gamma are shown in figure1, figure 2 and figure 3 respectively. Figure 4 shows the percentage (\%) of pixels passed the gamma criteria of 3\%-3mm using ImatriXX 2-D array system. In case of IMRT, the portal dosimetry mean values of area gamma, average gamma and maximum gamma were 1.01, 0.48 and 1.72 with standard deviation values of $0.38,0.05$ and 0.20 and for Rapid arc the corresponding values were 2.01, 0.48 and 1.72 with standard deviation of $0.54,0.07$ and 0.29 . With the ImatriXX 2-D array system, on an average $99.22 \%$ of the pixels 
passed the criteria of 3\%-3 mm with standard deviation of 0.58 for IMRT. For rapid arc, the average value was $97.14 \%$ with a standard deviation of 1.26 . The IMRT vs. rapid arc gamma results shows significant difference both in portal dosimetry as well as 2-D array measurements as shown in Table1.

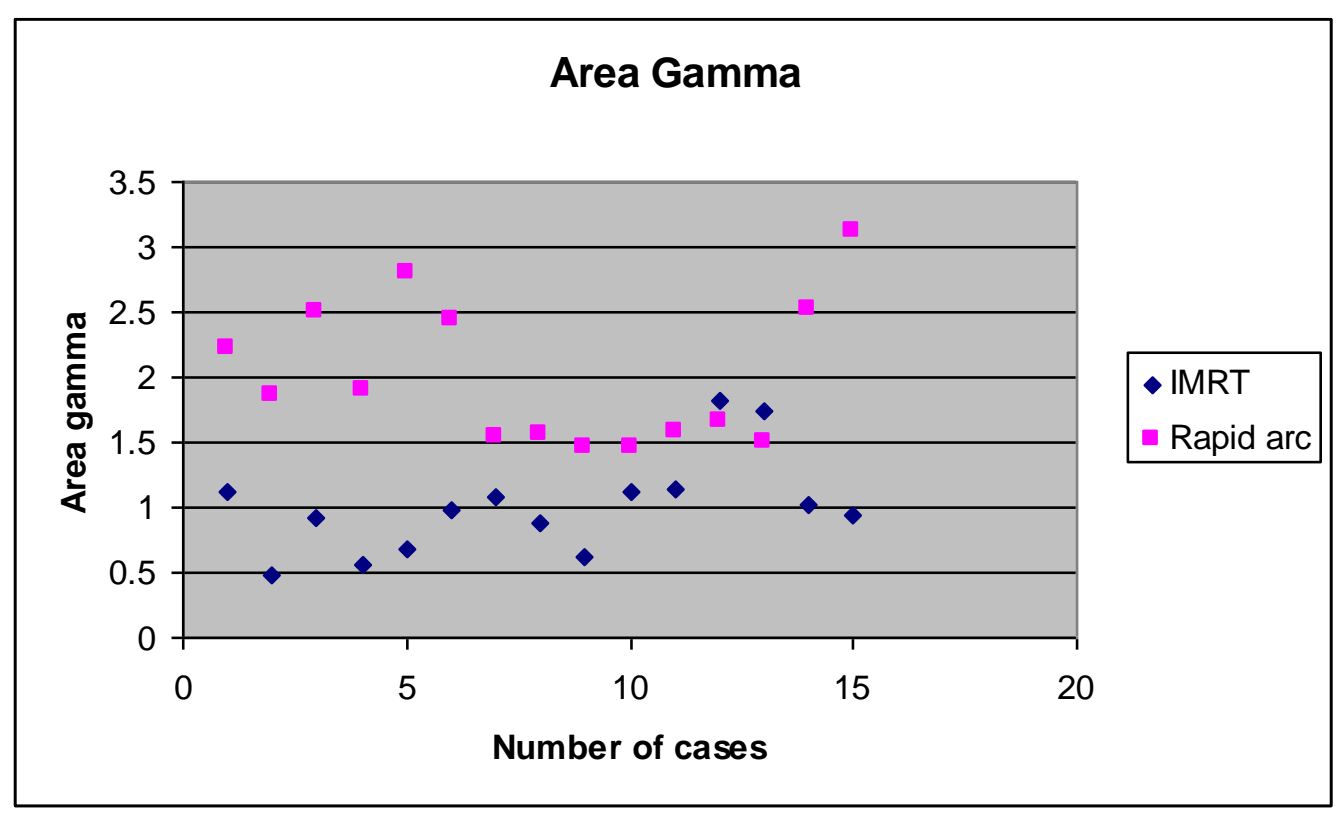

Figure1. Portal dosimetry area gamma results

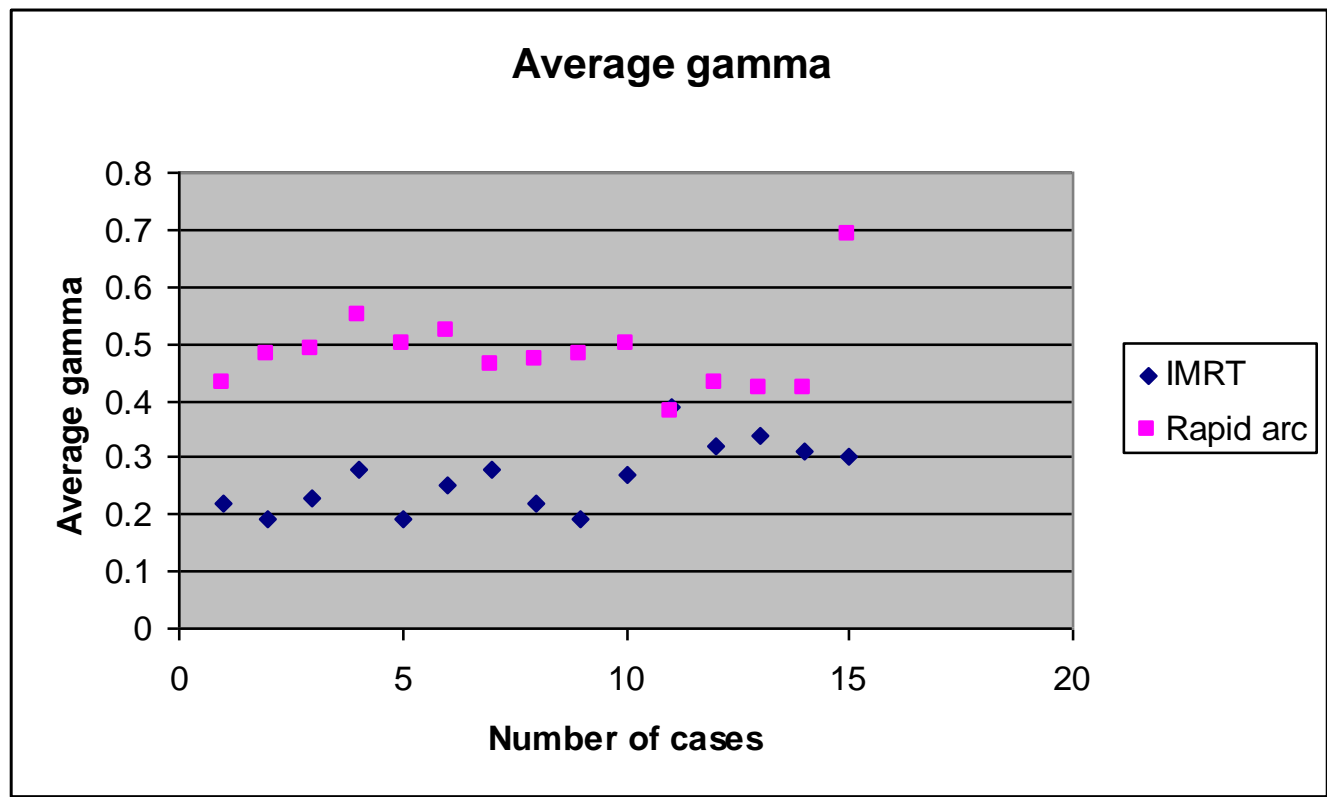

Figure2: Portal dosimetry average gamma results 


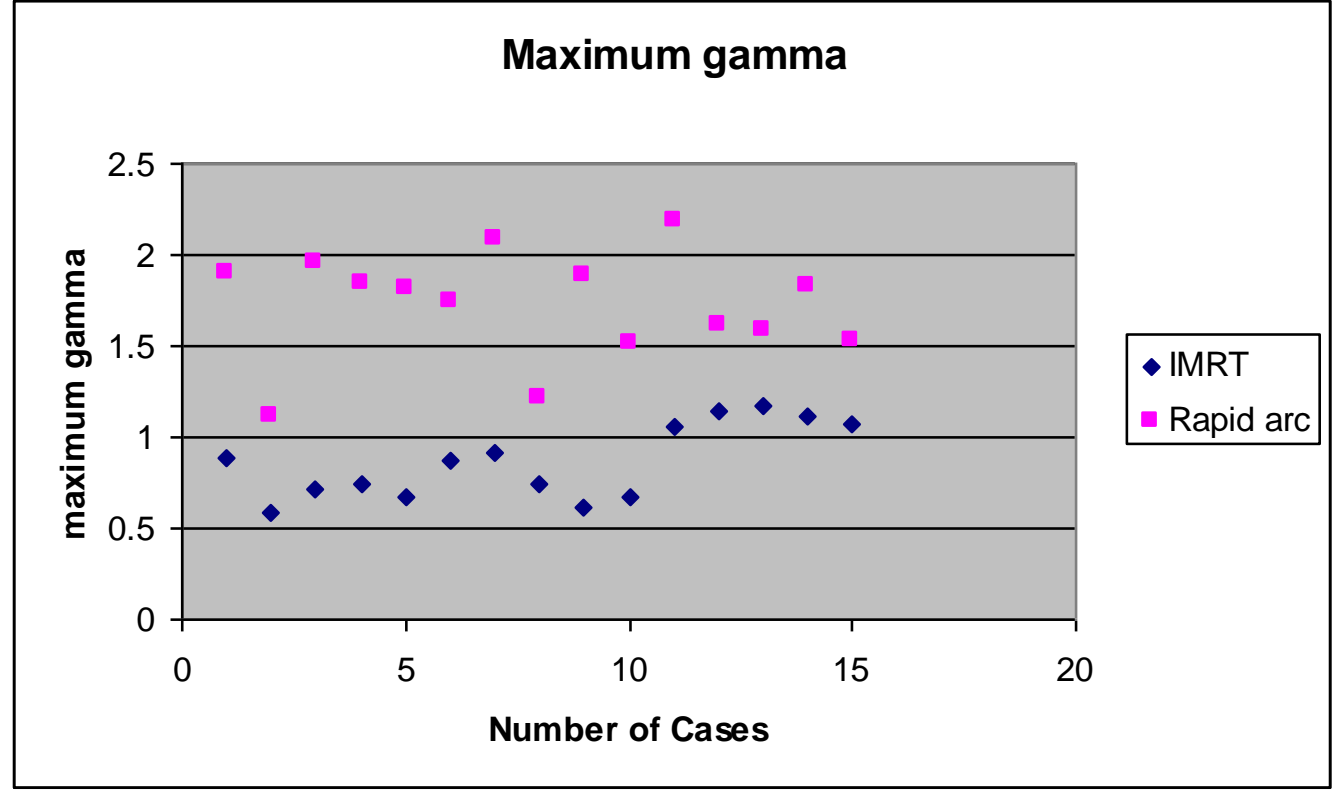

Figure 3: portal dosimetry maximum gamma results

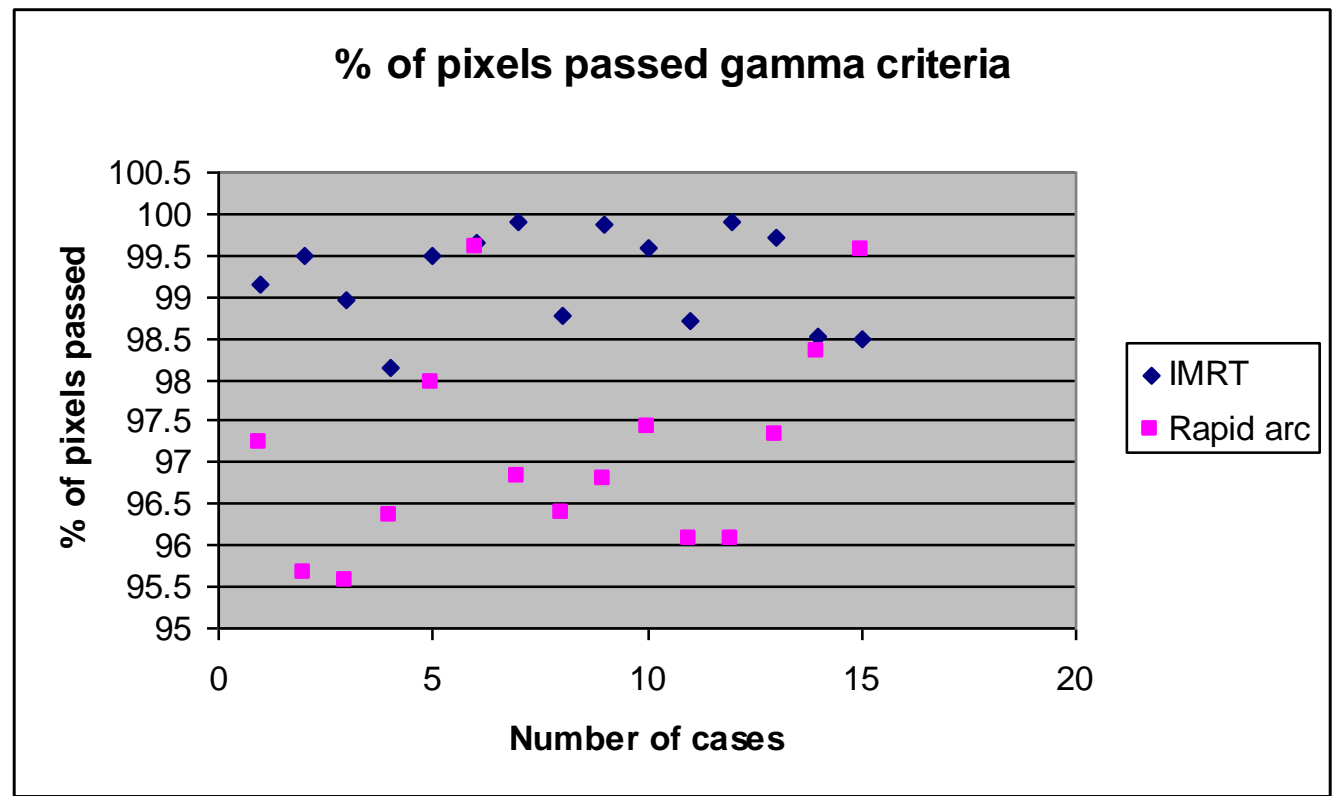

Figure4: ImatriXX 2-D array gamma results.

\begin{tabular}{|c|c|c|c|c|}
\hline & $\begin{array}{c}\text { Area } \gamma \geq 1 \% \\
\text { in portal } \\
\text { dosimetry }\end{array}$ & $\begin{array}{c}\text { Average } \gamma \text { in } \\
\text { portal dosimetry }\end{array}$ & $\begin{array}{c}\text { Max } \gamma \text { in } \\
\text { portal } \\
\text { dosimetry }\end{array}$ & $\begin{array}{c}\text { \% of pixels passed } \\
\text { in ImatriXX 2-D } \\
\text { array system }\end{array}$ \\
\hline IMRT vs. Rapid arc & $1.01+/-0.38$ & $0.86+/-0.20$ & \\
(Average and standard & vs. $2.01+/-$ & $0.26+/-0.05$ vs. & vs. $1.72+/-$ & $99.22+/-0.58$ vs. \\
deviation in QA results) & 0.54 & $0.48+/-0.07$ & 0.29 & $97.14+/-1.26$ \\
\hline P-value & 0.00012 & 0.000 & 0.000 & 0.000 \\
(IMRT vs. Rapid arc) & & & & \\
\hline
\end{tabular}

Table1. Average value, standard deviation and p-value of patient specific QA test results using portal dosimetry and ImatriXX 2-D array system.

\section{DISCUSSION}

One of the major concerns in a busy radiotherapy department is the time consumption for the quality assurance of individual patient plan. An easy to perform and reliable QA device plays an important role to 
reduce the labor and time consumption of medical physicists in radiation oncology. Many studies were published regarding the use of 2- D array detectors and aSi1000 EPIDs for quality assurance of IMRT and rapid arc patient specific plans (12-15). This study provides comparison results of patient specific IMRT and rapid arc plans using aSi1000 portal dosimetry and ImatriXX 2-D array system. The results of IMRT vs. rapid arc showed significant difference in passing rates of area gamma, average gamma, maximum gamma and $\%$ of pixels passed the criteria of $3 \%-3 \mathrm{~mm}$. While comparing the portal dosimetry and ImatriXX 2-D array system, the results are more consistent in portal dosimetry than ImatriXX 2-D array system. Both the system found to be equally good for IMRT and rapid arc patient specific QA. But while considering the workload of the department and labor oriented, the portal dosimetry system is less time consuming and easy for the set up. Individual field verification also found to be easier in portal dosimetry system in case of IMRT.

\section{CONCLUSION}

The study results showed that portal dosimetry as well as ImatriXX 2-D array systems can be use for patient specific QA measurements for IMRT and rapid arc. Compared to ImatriXX 2-D array system, the portal dosimetry values were more consistent. Significant differences were observed in comparison of IMRT and rapid arc gamma results. The portal dosimetry QA is less time consuming and easy to perform.

\section{REFERENCES}

[1] T. Wiezorek, M. Banz Nschwedas, D. Georg and T. G. Wendt, "Dosimetric quality assurance for intensity-modulated radiotherapy feasibility study for a filmless approach," Strahlenther. Oncol. , 181: 468-74, 2005.

[2] F. Branci Buonamici, L. Marrazzo and S. Russo, "An inter comparison between film dosimetry and diode matrix for IMRT quality assurance," Med. Phys., 34: 1372-1379, 2007.

[3] G. J. Budgell, Q. Zhang, R. J. Trouncer and R. I. Mackay, "Improving IMRT quality control efficiency using an amorphous silicon electronic portal imager," Med. Phys., 32: 3267-78, 2005.

[4] P. Winkler, A. Hefner and D. Georg, "Dose- response characteristics of an amorphous silicon EPID," Med. Phys., 32: 3095-105, 2005.

[5] E. E. Grein, R. Lee and K. Luchka, "An investigation of a new amorphous silicon electronic portal imaging device for transit dosimetry," Med. Phys., 29: 2262-8, 2002.

[6] P. B. Greer, C. C and Popescu, "Dosimetric properties of an amorphous silicon electronic portal imaging device for verification of dynamic intensity modulated radiation therapy," Med. Phys., 30: 1618-27, 2003.

[7] E Spezi , A.L Angelini, F Romani and A. Ferri, "Characterization of a 2D ion chamber array for the verification of radiotherapy treatments," Phys. Med. Biol., 50: 3361-3373, 2005.

[8] B. V. Elsevier, "Evaluation of a 2D diode array for IMRT quality assurance," Radiother. Oncol., 70: 199206, 2009.

[9] N. Agazaryan, D. S. Timothy and J. J. Demarco, "Patient specific quality assurance for the delivery of intensity modulated radiotherapy," J. Appl. Clin. Med. Phys., 4:, 2003.

[10] C. D. Wagter, "The ideal dosimeter for intensity modulated radiation therapy (IMRT): what is required?" Jr. Phys., 3: 4-8,2004.

[11] W. V. Elmpt, L. Mcdermott, S. Nijsten, M. wending, et al, "A literature review of electronic portal imaging for radiotherapy dosimetry," Radiother. oncol., 88: 289-309, 2008.

[12] D. Wagner and Hilke Vorwerk "Two years experience with quality assurance protocol for patient related Rapid Arc treatment plan verification using a two dimensional ionization chamber array." Radiat Oncol., 6: 21,2011

[13] A. Fogliata, , A Clivio, P. Fenoglietto, J. Hrbacek, et al, "Quality assurance of RapidArc in clinical practice using portal dosimetry” British Journal of Radiology, 84: 534-545, 2011.

[14] V. Chandraraj, S. Stathakis, R. Manickam, C. Esquivel' et al, "Comparison of four commercial devices for RapidArc and sliding window IMRT QA" Journal of Applied Clinical Medical Physics, 12, 2011.

[15] K. Murthy, " Patient-specific quality assurance of RapidArc treatments: Portal prediction dosimetry compared with phantom studies" Biomed Imaging Interv J., 8(4):e28, 2012. 